\section{A Suboptimal N-Step-Ahead Cautious Controller for Adaptive Control Applications}

\section{Jianjun Shi ${ }^{1}$ and Daniel W. Apley ${ }^{2}$}

If there is little a priori knowledge of the plant parameters, the performance of certainty equivalence based adaptive controllers during the transient period, before the parameter estimates have converged, is usually less than adequate. This paper introduces a new suboptimal loss function with an $N$-step-ahead prediction horizon, similar in principle to standard predictive control loss functions, for adaptive control applications. However, the new loss function is modified so that the resulting control law possesses caution in the sense that the uncertainty in the parameter estimates is taken into account automatically. As such, the cautious controller achieves more robust performance than the corresponding certainty equivalence controller during the transient period when there is large uncertainty in the parameter estimates. The new cautious controller has a closed-form solution that involves only slightly more computational expense than the corresponding certainty equivalence predictive controller.

\section{Introduction}

Certainty equivalence ( $\mathrm{CE}$ ) is a widely used principle in the design of adaptive controllers and often results in a reasonably effective and easily implemented control law. However, if there is little a priori knowledge of the plant parameters, the performance of the CE-based adaptive controller during the transient period may be less than adequate. This results from the fact that under the $C E$ principle the estimated parameters are treated as the true parameters, even though they may be far from their true values before they have had a chance to converge. A theoretically attractive alternative is to exactly minimize a stochastic quadratic loss function, which results in an optimal input that possess both caution and probing (Astrom and Wittenmark, 1995). An adaptive controller is probing if it attempts to excite the system to improve the parameter estimation, in addition to driving the system to a desired state. A controller is cautious if the control input is a function of the covariance of the parameter estimates and takes more cautious (i.e. less agressive) action if the parameter uncertainties are large. These properties are discussed in detail in Jacobs and Patchell (1972), Bar-Shalom and Tse (1974), Sternby (1979), and Bar-Shalom (1981).

The advantage of the cautious and probing optimal stochastic adaptive controller is that uncertainties in the parameter estimates, which can be particularly large during the transient period, are dealt with in an "optimal" manner. Unfortunately, for all but the simplest of situations the problem is far too complicated to solve exactly. Consequently, numerous researchers (Tse and Bar-Shalom, 1973; Ku and Athans, 1973; Hughes and Jacobs, 1973; Wittenmark, 1975; Sternby, 1976; Milito et al., 1980; Mookerjee and Bar Shalom, 1989) have attempted to modify the optimal control loss function and/or introduce simplifying assumptions so that the re-

\footnotetext{
'Department of Industrial and Operations Engineering, The University of Michigan, Ann Arbor, MI 48109-21.17.

${ }^{2}$ Department of Industrial Engineering, Texas A\&M University, College Station, TX 77843-3131.

Contributed by the Dynamic Systems and Control Division of THE AMERICAN Society of Mechanical Engineers. Manuscript received by the Dynamic Systems and Control Division March 4, 1994. Associate Technical Editor: N. Sadegh.
}

sulting control law is implementable, while retaining some elements of caution and probing. Many of these techniques have no closed-form solution and are too complicated to practically implement (Tse and Bar-Shalom, 1973; Ku and Athans, 1973; Mookerjee and Bar Shalom, 1989). Sternby (1976) develops a dual controller for the specific case that the plant is a one-dimensional four-state Markov chain. Hughes and Jacobs (1973), Wittenmark (1975), and Milito et al. (1980) restrict the loss function to a single-step prediction horizon, and thus lose the flexibility of multi-step loss functions popular in predictive and LQ control strategies.

The purpose of this paper is to introduce a new suboptimal $\mathrm{N}$-step-ahead loss function for adaptive control applications. In addition to having a relatively simple closed-form control law and no restrictions on the length of the prediction horizon, the resulting controller possesses caution in the sense that the control input is explicitly a function of the parameter estimation error covariance information. As such, the performance during the transient period when the parameter estimates are less accurate is improved over the corresponding $\mathrm{CE}$ controller. In Section 2 the new suboptimal $\mathrm{N}$-step-ahead loss function is introduced and the cautious control law is derived. Monte Carlo simulation results comparing the cautious controller with the corresponding $\mathrm{CE}$ controller are presented in Section 3.

\section{The Suboptimal N-Step-Ahead Cautious Control- ler}

Consider the single-input single-output, time-varying, linear stochastic system

$$
\begin{gathered}
z(k+1)=A(k) z(k)+b(k) u(k)+w(k) \\
y(k)=C z(k)+v(k),
\end{gathered}
$$

where $z(k) \in \mathbb{R}^{n}, y(k) \in R^{1}, w(k) \in R^{n}, v(k) \in R^{1}$, and $u(k)$ $\in R^{1}$ are the state, output, system noise, observation noise, and input, respectively. $k$ is a time index. $z(0)$ and $w(k), v(k+1)$ $(k=0,1,2 \ldots)$ are assumed to be independent Guassian random vectors with zero mean and known covariance matrices. Let the system be represented in observable canonical form such that

$$
\begin{aligned}
& A(k)=\left\{\begin{array}{ccccc}
a_{1}(k) & 1 & 0 & \cdots & 0 \\
a_{2}(k) & 0 & 1 & \cdots & 0 \\
\vdots & & & \ddots & \\
a_{n-1}(k) & 0 & 0 & & 1 \\
a_{n}(k) & 0 & 0 & \cdots & 0
\end{array}\right\}, \\
& b(k)=\left\{\begin{array}{c}
b_{1}(k) \\
b_{2}(k) \\
\vdots \\
b_{n-1}(k) \\
b_{n}(k)
\end{array}\right\}, \quad \text { and } C=\left[\begin{array}{lll}
1 & 0 \ldots 0
\end{array}\right] \text {. }
\end{aligned}
$$

Define $a(k)=\left[a_{1}(k) a_{2}(k) \ldots a_{n}(k)\right]^{T}$, and assume the unknown parameters of the system are modeled by the GaussMarkov process

$$
\left[\begin{array}{l}
a(k+1) \\
b(k+1)
\end{array}\right]=\left[\begin{array}{l}
a(k) \\
b(k)
\end{array}\right]+\gamma(k),
$$

where $\gamma(k)$ is a zero-mean uncorrelated Gaussian process with known covariance matrix.

By defining the augmented state vector $x(k) \equiv\left[z^{T}(k) a^{T}(k)\right.$ $\left.b^{T}(k)\right]^{T},(1)$ can be written as the nonlinear stochastic system 


$$
x(k+1)=f(x(k))+g(x(k)) u(k)+\left[\begin{array}{c}
w(k) \\
\gamma(k)
\end{array}\right], \text { where }
$$

$$
f(x(k)) \equiv\left\{\begin{array}{c}
A(k) z(k) \\
a(k) \\
b(k)
\end{array}\right\} \text { and } g(x(k)) \equiv\left\{\begin{array}{c}
b(k) \\
0 \\
0
\end{array}\right\}
$$

The representation (2), including the Gauss-Markov model for the unknown parameters, is commonly used when posing optimal stochastic adaptive control problems or when using an extended Kalman filter (EKF) to estimate the unknown parameters of the system. Throughout the remainder of the paper it is assumed that an EKF is used to provide estimates of the augmented state $(z(k)$, as well as the unknown parameters) and the state estimation error covariance matrix, defined as

$$
\begin{gathered}
\hat{x}(k) \equiv E\left[x(k) \mid Y^{k}, U^{k-1}\right] \quad \text { and } \\
P(k) \equiv E\left[[x(k)-\hat{x}(k)][x(k)-\hat{x}(k)]^{T} \mid Y^{k}, U^{k-1}\right] .
\end{gathered}
$$

Here, $U^{k-1} \equiv\{u(0), u(1), \ldots, u(k-1)\}, Y^{k} \equiv\{y(1), y(2)$, $\ldots, y(k)\}$, and $E[\alpha \mid \beta]$ denotes the conditional expectation of random variable $\alpha$, conditioned on the sigma-algebra generated by random variables $\beta$. It is straightforward (Ljung and Soderstrom, 1983) to design an EKF for (2).

Assume that at time $k$ the control sequence $U^{k-1}$ has been applied to the system and the observation sequence $Y^{k}$ has been obtained. Introduce the loss function

$$
\begin{gathered}
j(k) \equiv \frac{1}{2} E\left[\sum_{i=1}^{N} \hat{x}^{T}(k+i \mid k) Q \hat{x}(k+i \mid k)\right. \\
\left.+r u^{2}(k+i-1) \mid Y^{k}, U^{k-1}\right] \\
Q \equiv\left\{\begin{array}{ccc}
Q_{z} & \mathbf{0} & \mathbf{0} \\
\mathbf{0} & \mathbf{0} & \mathbf{0} \\
\mathbf{0} & \mathbf{0} & \mathbf{0}
\end{array}\right\}
\end{gathered}
$$

where $Q_{2}$ is a positive-semidefinite $n \times n$ matrix, $N$ is the prediction horizon, $r \geq 0$ is a scalar input weighting factor, and 0 denotes an $n \times n$ matrix of zeros. For the remainder of the paper, the notation 0 will refer to either an $n$-length vector or $n \times n$ matrix of zeros, which will be clear from the context. I will denote the $n \times n$ identity matrix. $\hat{x}(k+i \mid k)$ is the $i$-stepahead prediction of the state at time $k$.

The main difference between (3) and standard predictive control loss functions lies in the definition of $\hat{x}(k+i \mid k)$. For $i=1,2, \ldots, N$, define

$$
\hat{x}(k+i \mid k) \equiv E\left[x(k+i) \mid Y^{k}, U^{k-1}, \tilde{x}(k)\right],
$$

where $\tilde{x}(k) \equiv x(k)-\hat{x}(k)$ is the state estimation error at time $k$. Thus, $\hat{x}(k+i \mid k)$ is allowed to be a function of the unknown random variable $\tilde{x}(k)$, which accounts for the need for the conditional expectation in (3). Note that no such expectation is needed in standard predictive control loss functions, where the $i$-step-ahead prediction of the state is not allowed to depend on any unknown random variables. The dependence of $\hat{x}(k+$ $i \mid k)$ on $\tilde{x}(k)$ is essentially what results in the cautious property of the proposed controller, since, after making appropriate substitutions and taking the expectation in $(3), P(k)$ will be present in the control law.

At time $k, u(k)$ is selected to minimize $J(k)$ subject to the constraint $u(k+i)=0$ for $i=1,2, \ldots, N-1 . u(k)$ is then applied to the system, and the process is repeated at time $k+$ 1 . Thus, the control strategy is receding horizon suboptimal control. The constraint $u(k+i)=0$ for $i=1,2, \ldots, N-1$ is a commonly employed constraint in predictive control where the objective is to control the system about a zero setpoint (De Keyser et al., 1988). The interpretation is that one assumes $u(k)$, and no other control action, will be applied to bring the system back to the desired value within the prediction horizon.

Assuming the unknown parameters vary slowly, so that over the prediction horizon (2) can be linearized about the parameter estimates at time $k,(2)$ can be approximated by

$$
\begin{aligned}
& x(k+i) \cong F(k) x(k+i-1)+[g(\hat{x}(k)) \\
&+G(k)(x(k+i-1)-\hat{x}(k))] u(k+i-1) \\
&+D(k)+\left[\begin{array}{c}
w(k+i-1) \\
\gamma(k+i-1)
\end{array}\right],
\end{aligned}
$$

where

$$
\begin{gathered}
\left.F(k) \equiv \frac{\partial f(\xi)}{\partial \xi}\right|_{\xi=\hat{x}(k)}=\left[\begin{array}{ccc}
\hat{A}(k) & \mathbf{I} y(k) & \mathbf{0} \\
\mathbf{0} & \mathbf{I} & \mathbf{0} \\
\mathbf{0} & \mathbf{0} & \mathbf{I}
\end{array}\right] \\
\left.G(k) \equiv \frac{\partial g(\xi)}{\partial \xi}\right|_{\xi=\hat{x}(k)}=\left[\begin{array}{lll}
\mathbf{0} & \mathbf{0} & \mathbf{I} \\
\mathbf{0} & \mathbf{0} & \mathbf{0} \\
\mathbf{0} & \mathbf{0} & \mathbf{0}
\end{array}\right], \\
g(\hat{x}(k))=\left[\begin{array}{c}
\hat{b}(k) \\
\mathbf{0} \\
\mathbf{0}
\end{array}\right], \quad \text { and } \\
D(k) \equiv f(\hat{x}(k))-F(k) \hat{x}(k)=\left[\begin{array}{c}
-\hat{a}(k) y(k) \\
\mathbf{0} \\
\mathbf{0}
\end{array}\right] .
\end{gathered}
$$

Here, $\hat{x}(k)$ has been partitioned as $\left[\hat{z}^{T}(k) \hat{a}^{T}(k) \hat{b}^{T}(k)\right]^{T}$, and $\hat{A}(k)$ denotes $A(k)$ with its parameters replaced by their estimates.

Taking the conditional expectation of (4) gives

$$
\hat{x}(k+i \mid k)=\left\{\begin{aligned}
F(k) \hat{x}(k)+F(k) \tilde{x}(k)+[g(\hat{x}(k)) \\
+G(k) \hat{x}(k)] u(k)+D(k) \quad: i=1 . \\
F(k) \hat{x}(k+i-1 \mid k)+D(k) \quad: i \geq 2
\end{aligned}\right.
$$

The expression for $i=1$ results from the fact that $E\left[x(k) \mid Y^{k}\right.$, $\left.U^{k-1}, \tilde{x}(k)\right]=x(k)=\hat{x}(k)+\tilde{x}(k)$. Solving explicitly for $i=$ $1,2, \ldots, N$ gives

$$
\begin{array}{r}
\hat{x}(k+i \mid k)=F^{i}(k) \hat{x}(k)+F^{i}(k) \tilde{x}(k)+F^{i-1}(k)[g(\hat{x}(k)) \\
+G(k) \tilde{x}(k)] u(k)+\sum_{j=0}^{i-1} F^{j}(k) D(k) .
\end{array}
$$

Substituting (5) into (3) results in $J(k)$ being a quadratic function of $u(k)$. Thus, the optimal input can be found by setting $\partial J(k) / \partial u(k)=0$ and solving for $u(k)$. Noting that $u(k)$ is required to be a measureable function of $\left\{U^{k-1}, Y^{k}\right\}$ and that $E\left[\tilde{x}(k) \mid Y^{k}, U^{k-1}\right]=\mathbf{0}$, it can be shown that the optimal input is given by

$$
\begin{gathered}
u(k)=-\left\{\sum_{i=1}^{N}\left(h_{1, i}+E\left[\tilde{x}^{T}(k) H_{3, i} \tilde{x}(k) \mid Y^{k}, U^{k-1}\right]\right)+r\right\}^{-1} \\
\times\left\{\sum_{i=1}^{N}\left(h_{2, i}+E\left[\tilde{x}^{T}(k) H_{4, i} \tilde{x}(k) \mid Y^{k}, U^{k-1}\right]\right)\right\}, \text { where } \\
h_{1, i} \equiv\left[F^{i-1}(k) g(\hat{x}(k))\right]^{T} Q F^{i-1}(k) g(\hat{x}(k)) \\
=\hat{b}^{T}(k)\left[\hat{A}^{i-1}(k)\right]^{T} Q_{z} \hat{A}^{i-1}(k) \hat{b}(k) \\
h_{2, i} \equiv\left[F^{i-1}(k) g(\hat{x}(k))\right]^{T} Q\left[F^{i}(k) \hat{x}(k)+\sum_{j=0}^{i-1} F^{j}(k) D(k)\right] \\
=\hat{b}^{T}(k)\left[\hat{A}^{i-1}(k)\right]^{T} Q_{2} \hat{A}^{i}(k) \hat{z}(k)
\end{gathered}
$$




$$
\begin{aligned}
H_{3, i} & \equiv\left\{F^{i-1}(k) G(k)\right]^{T} Q\left[F^{i-1}(k) G(k)\right] \\
& =\left[\begin{array}{ccc}
\mathbf{0} & \mathbf{0} & \mathbf{0} \\
\mathbf{0} & \mathbf{0} & \mathbf{0} \\
\mathbf{0} & \mathbf{0} & {\left[\hat{A}^{i-1}(k)\right]^{T} Q_{2} \hat{A}^{i-1}(k)}
\end{array}\right], \text { and } \quad(9) \\
H_{4, i} & \equiv\left[F^{i-1}(k) G(k)\right]^{T} Q F^{i}(k) \\
& =\left[\begin{array}{ccc}
\mathbf{0} & \mathbf{0} & \mathbf{0} \\
\mathbf{0} & \mathbf{0} & \mathbf{0} \\
{\left[\hat{A}^{i-1}(k)\right]^{T} Q_{z} \hat{A}^{i}(k)} & {\left[\hat{A}^{i-1}(k)\right]^{T} Q_{z} \sum_{j=0}^{i-1} \hat{A}^{j}(k) y(k)} & \mathbf{0}
\end{array}\right] .
\end{aligned}
$$

(6) through (10) follow via straightforward but tedious algebra, the details of which have been omitted.

(6) can be further simplified by noting that, for an $n$-lenth random vector $x$ and an $n \times n$ deterministic matrix $M, E\left[x^{T} M x\right]$ $=\operatorname{tr}\left(E\left[x x^{T}\right] M\right)$, where $\operatorname{tr}(\cdot)$ denotes the trace of a matrix. Consequently, (6) can be rewritten as

$$
\begin{gathered}
u(k) \\
=-\left\{\sum_{i=1}^{N}\left(h_{1, i}+h_{3, i}\right)+r\right\}^{-1}\left\{\sum_{i=1}^{N}\left(h_{2, i}+h_{4, i}\right)\right\}, \text { where } \\
h_{3, i} \equiv E\left[\tilde{x}^{T}(k) H_{3, i} \tilde{x}(k) \mid Y^{k}, U^{k-1}\right]=\operatorname{tr}\left(P(k) H_{3, i}\right) \\
=\operatorname{tr}\left(P_{b}(k)\left[\hat{A}^{i-1}(k)\right]^{T} Q_{2} \hat{A}^{i-1}(k)\right) \\
h_{4, i} \equiv E\left[\hat{x}^{T}(k) H_{4, i} \tilde{x}(k) \mid Y^{k}, U^{k-1}\right] \\
=\operatorname{tr}\left(P(k) H_{4, i}\right)=\operatorname{tr}\left(P_{z b}(k)\left[\hat{A}^{i-1}(k)\right]^{T} Q_{z} \hat{A}^{i}(k)\right) \\
+\operatorname{tr}\left(P_{a b}(k)\left[\hat{A}^{i-1}(k)\right]^{T} Q_{z} \sum_{j=0}^{i-1} \hat{A}^{j}(k) y(k)\right)
\end{gathered}
$$

Here, $P(k)$ has been partitioned into $n \times n$ blocks as $P(k)=$

$$
\left[\begin{array}{lll}
P_{z}(k) & P_{z a}(k) & P_{z b}(k) \\
P_{z a}^{T}(k) & P_{a}(k) & P_{a b}(k) \\
P_{z b}^{T}(k) & P_{a b}^{T}(k) & P_{b}(k)
\end{array}\right]
$$

Remark 2.I. The control law (11) is cautious in the sense that $u(k)$ is a function of not only the instantaneous state estimate but also its error covariance information through the terms $h_{3, i}$ and $h_{4, i}$, i.e. $u(k)=u(\hat{x}(k), P(k))$. The term $h_{3, i} \equiv$ $E\left[\tilde{x}^{T}(k) H_{3, i} \tilde{x}(k) \mid Y^{k}, U^{k-1}\right] \geq 0$ is contained in the denominator, which has the effect of reducing the input magnitude when $P(k)$ is large. On the other hand, if $P(k)$ is small, the state estimation error is neglected and the cautious control law approaches that of CE control (see Remark 2.2 below). This cautious property, which automatically adjusts the control action according to the uncertainty in the state and parameter estimation, is a key feature of the control law (11). As illustrated in the following section, caution is especially important during the transient period of adaptive control when there can be large uncertainties in the state and parameter estimation.

Remark 2.2. If CE were enforced, the corresponding CE adaptive predictive control law could be obtained by setting the state estimation error $\tilde{x}(k)$ equal to zero in (5) or, equivalently, setting $P(k)$ equal to zero in (12) and (13). The resulting CE control law would then be

$$
u_{C E}(k)=-\left\{\sum_{i=1}^{N} h_{1, i}+r\right\}^{-1}\left\{\sum_{i=1}^{N} h_{2, i}\right\} \text {. }
$$

Remark 2.3. (11) provides a closed-form expression for the cautious control law, with computational expense that is not greatly increased over that for the CE control law of (14). The majority of the computational expense in calculating the additional quantities $h_{3, i}$ and $h_{4, i}$ is in calculating the terms $\left[\hat{A}^{i-1}(k)\right]^{T} Q_{z} \hat{A}^{i-1}(k)$,

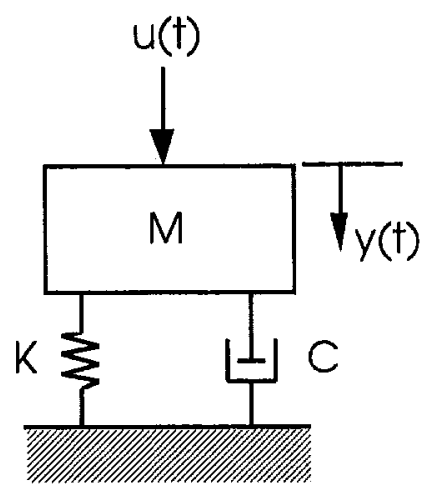

Fig. 1 Mass/spring/damper system used in the simulations

which are also required in calculating $h_{1, i}$ and $h_{2, i}$ in the CE control law. This is one of the major advantages of the proposed cautious controller over the existing multi-step horizon cautious controllers of Tse and Bar-Shalom (1973) and Ku and Athans (1973), which have no closed form solution and are computationally expensive. Also, the arbitrary time horizon $N$ in the cost function of (3) allows greater flexibility in controller design than in the cautious control approaches of Hughes and Jacobs (1973), Wittenmark (1975), Milito et al. (1980), and Mookerjee and Bar Shalom (1989), where the prediction horizon is restricted to only one timestep.

\section{Simulation Results}

The purpose of the simulations is to illustrate the cautious properties of the cautious control law (11) by comparing its performance during the transient period, before the parameter estimates have converged, with that of the corresponding $\mathrm{CE}$ control law (14). The system studied is the second order mass/ spring/damper system illustrated in Fig. 1, where the parameters $M$ (mass), $K$ ( spring constant), and $C$ (damping constant) are unknown and must be estimated adaptively. $y(t)$ and $u(t)$ are the displacement and force, respectively, at time $t$. Using numerical values of $M=5 \mathrm{~kg}, K=50 \mathrm{~N} / \mathrm{cm}, C=32 \mathrm{Ns} / \mathrm{m}$ and a sampling rate of $0.02 \mathrm{~s}$, the observable canonical form of the discrete time system becomes

$$
\begin{gathered}
z(k+1)=\left[\begin{array}{ll}
a_{1} & 1 \\
a_{2} & 0
\end{array}\right] z(k)+\left[\begin{array}{l}
b_{1} \\
b_{2}
\end{array}\right] u(k)+w(k), \\
y(k)=\left[\begin{array}{ll}
1 & 0
\end{array}\right] z(k), \text { where }
\end{gathered}
$$

$a_{1}=1.517, a_{2}=-0.880, b_{1}=0.371$, and $b_{2}=0.355$. For scaling purposes, $y(k)$ is measured in units of $\mathrm{cm}$ and $u(k)$ in units of $100 \mathrm{~N}$. Zero-mean Gaussian process noise $w(k)$, with covariance matrix $0.25 \mathrm{I}$, has been added to the system, and it is assumed there is no measurement noise.

In order to implement the cautious controller for the general system (1), it is necessary to select the controller parameters $N, r$, and $Q_{z}$ and the Kalman filter parameters $P(0), \hat{z}(0), \hat{a}(0)$, and $\hat{b}(0)$. Values for $N, r$, and $Q_{z}$ should be chosen based on the control objective. In general, the values for $P(0), \hat{z}(0)$, $\hat{a}(0)$, and $\hat{b}(0)$ should be chosen based on any a priori information available. A common practice in extended Kalman filtering is to set $P_{z a}(0)=P_{z b}(0)=P_{a b}(0)=0$ and to set $P_{z}(0), P_{a}(0)$ and $P_{b}(0)$ to diagonal matrices. Using the notation $P_{z}(0)=$ $\operatorname{diag}\left\{\sigma_{z_{1}}^{2}, \sigma_{z_{2}}^{2}, \ldots, \sigma_{z_{n}}^{2}\right\}, P_{a}(0)=\operatorname{diag}\left\{\sigma_{a_{1}}^{2}, \sigma_{a_{2}}^{2}, \ldots, \sigma_{a_{n}}^{2}\right\}$, and $P_{b}(0)=\operatorname{diag}\left\{\sigma_{b_{1}}^{2}, \sigma_{b_{2}}^{2}, \ldots, \sigma_{b_{n}}^{2}\right\}$, the diagonal elements should be set to reflect, as accurately as possible, the initial uncertainty in the state and parameter estimates. Based on the authors' experience, it is recommended that the $\sigma$ 's be specified so that, with reasonable confidence, the true value of the corre- 


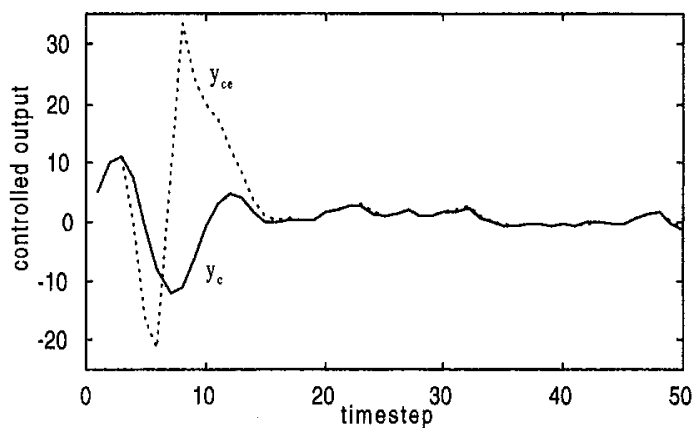

Fig. 2 Comparison of the controlled output using cautious control $\left(y_{c}\right)$ and CE control $\left(y_{c \theta}\right)$ with $b_{0}=0.1$ and $\sigma_{b}=0.7$

sponding component of the state or parameter is known to lie within the range $\pm \sigma$ of its initial guess.

For all simulations the following values were used. The controller parameters were $N=5, r=0.5$ and $Q_{z}=\left[\begin{array}{ll}1 & 0 \\ 0 & 0\end{array}\right]$. The system state was given initial conditions $z(0)=\left[\begin{array}{ll}5 & 3\end{array}\right]^{T}$. Initial guesses for the state and parameters were $\hat{z}(0)=\left[\begin{array}{ll}0 & 0\end{array}\right]^{T}, \hat{a}(0)$ $=\left[\begin{array}{ll}0 & 0\end{array}\right]^{T}$, and $\hat{b}(0)=\left[\begin{array}{ll}b_{0} & b_{0}\end{array}\right]^{T}$, where $b_{0}$ was varied between -0.5 and 1.0. Values used for the assumed uncertainty in the initial guesses were $\sigma_{z_{1}}=\sigma_{z_{2}}=5.0, \sigma_{a_{1}}=1.5, \sigma_{a_{2}}=1.0$, and $\sigma_{b_{1}}=\sigma_{b_{2}}=\sigma_{b}$, where $\sigma_{b}$ was either 0.7 or 1.0 . In general, this represents a situation where there is very little a priori knowledge of the parameters.

Figures 2 and 3 show typical simulation results for $b_{0}=0.1$ and $\sigma_{b}=0.7$ for both the cautious control law (11) and the corresponding CE control law (14). Figure 2 shows the controlled output using the cautious control law $\left(y_{c}\right)$ and $C E$ control law $\left(y_{c e}\right)$, and Fig. 3 shows the corresponding inputs. With $\hat{b}(0)$ underestimated, during the initial timesteps when the parameter estimation has not yet converged and is still inaccurate, the CE control law calls for very large inputs. The result is a large temporary burst in the output, as shown in Fig. 2. In contrast, the cautious control law takes into account the uncertainties in the parameter estimates and acts much more conservatively during the transient period. $u_{c}$ remains at reasonable levels, and there is no large burst in $y_{c}$. After approximately 25 timesteps the parameter estimates converge to close to their true values, the elements of $P(k)$ that reflect the uncertainties in the parameter estimates become negligibly small, and the two control laws become nearly identical. Thus, the steady-state performance of the cautious control law and the $\mathrm{CE}$ control law are nearly identical.

In order to investigate the effects of caution and inaccuracies in the initial guesses for the parameters, a Monte Carlo simulation was used to compare the cautious and $\mathrm{CE}$ control laws as the value for $b_{0}$ varied between -0.5 and 1.0 . The criterion used to compare the control laws is the evaluated loss function (3) averaged over the transient period (the first 50 timesteps) of the simulation, i.e.

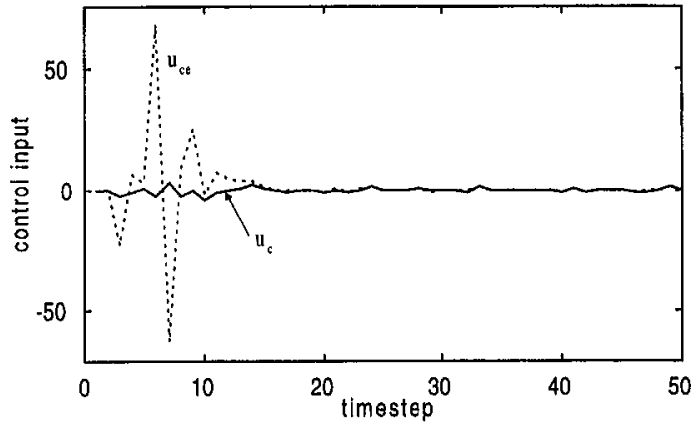

Fig. 3 Comparison of the control input using cautious control $\left(u_{c}\right)$ and CE control $\left(u_{c e}\right)$ with $b_{0}=0.1$ and $\sigma_{b}=0.7$. The output is shown in Fig. 2.

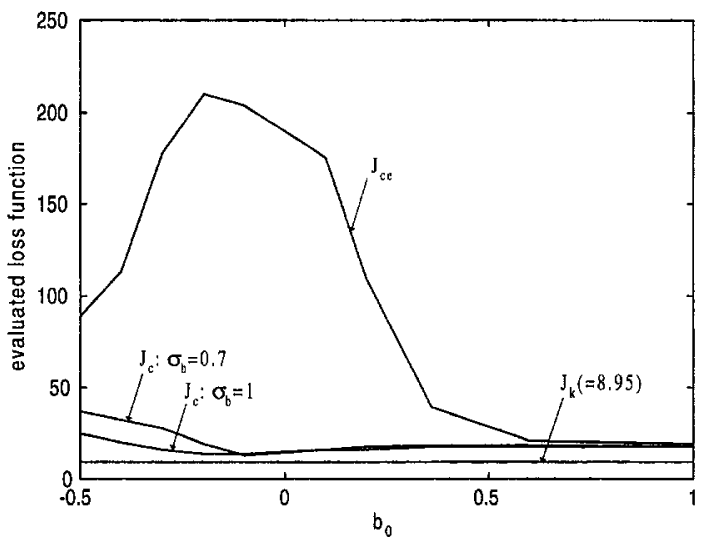

Fig. 4 Comparison of the performance criterion using CE control $\left(J_{c o}\right)$ cautious control $\left(J_{c}\right)$ with two different values for the initial measure of the parameter uncertainty, and the corresponding control law with the parameters known $\left(J_{k}\right)$. Values are plotted versus $b_{0}$.

$$
J_{m} \equiv \frac{1}{50} \sum_{k=1}^{50} x^{T}(k) Q x(k)+r u^{2}(k-1),
$$

where $m=$ ' $c$ ' indicates the cautious control law was used, $m$ $=$ ' $c e$ ' indicates the CE control law was used, and $m=$ ' $k$ ' indicates the true parameters were known and available to the controller. That is, $m=$ ' $k$ ' indicates the CE control law (14) was used with the estimated parameters replaced by their true values. $J_{k}$ serves as a benchmark for the minimum achievable loss function. For each control law and each value of $b_{0}, 1000$ Monte Carlo simulations were run to evaluate the performance criteria, the average of which is plotted in Fig. 4. $J_{c e}, J_{k}$, and $J_{c}$ with two different values of $\sigma_{b}$ are plotted versus $b_{0}$. Note that since $J_{k}$ uses the true parameters, as opposed to their estimates, it does not depend on the initial guess $b_{0}$.

Remark 3.1. It is apparent from Fig. 4 that the transient performance of the $\mathrm{CE}$ controller is highly sensitive to the initial guesses for the parameters $b_{1}$ (true value is 0.371 ) and $b_{2}$ (true value is 0.355 ). With $b_{1}$ and $b_{2}$ initially underestimated (e.g. $b_{0}=0.1$ ) or the sign reversed (e.g., $b_{0}=-0.3$ ), $J_{c e}$ increases markedly. Situations like this result in large initial bursts in the input and output, as illustrated in Figs. 2 and 3. In contrast, the cautious control law is far less sensitive to the initial guesses for the parameters. Assuming an initial uncertainty of $\sigma_{b}=0.7$, the cautious controller is robust with respect to errors in the initial guess until $b_{0}$ drops below approximately -0.1 , at which point $J_{c}$ begins to grow. By assuming a large initial uncertainty of $\sigma_{b}=1.0$, the robustness is extended to even smaller values of $b_{0}$. For example, with $b_{0}=-0.4\left(J_{c}=20.2\right)$ the cautious controller performs almost as well as if $b_{0}=0.36\left(J_{c}=17.5\right)$. It is interesting to note that for $b_{1}$ and $b_{2}$ initially overestimated (e.g. $b_{0}=1.0$ ), the $\mathrm{CE}$ controller performs similarly to the cautious controller. The explanation is that overestimating $b_{1}$ and $b_{2}$ overestimates the effect of the input and results in smaller input magnitudes, which is a form of caution.

Remark 3.2. The main performance advantage of the cautious controller over the $\mathrm{CE}$ controller occurs in situations where there is large uncertainty in the parameter estimates, in particular during the transient period before the estimates have had a change to converge. After the parameter estimates have converged, there is much less uncertainty in the system. $P_{z b}(k), P_{a b}(k)$, and $P_{b}(k)$ typically grow small enough that the terms $h_{3, i}$ and $h_{4, i}$ in the control law (11) are negligible, in which case the cautious control law approaches the CE control law. For all cases considered and all three control laws (cautious, $\mathrm{CE}$, and known parameters), the steady-state performance of the three controllers was virtually identical, never differing by more than $3 \%$. 


\section{Conclusions}

Through modification of a standard multi-step horizon predictive control loss function, a new suboptimal N-step-ahead cautious controller for adaptive control applications has been developed in this paper. The modification involves allowing the predicted value of the future state to be a function of the current error in the sate and parameter estimation. The control law that results is cautious in the sense that the control input is a function of $\boldsymbol{P}(k)$. If the uncertainty in the parameter estimation is large, elements of $P(k)$ will be large and the controller takes less aggressive action than if the uncertainty is small. The new controller is unique in the sense that it has a relatively simple closed-form solution that combines caution with the versatility of a multi-step horizon loss function. It has been demonstrated via Monte Carlo simulation that in the transient period, when the uncertainty in the parameter estimation is large, the cautious controller can achieve substantially better performance than the corresponding $\mathrm{CE}$ adaptive predictive controller.

\section{References}

Astrom, K. J., and Wittenmark, B., 1995, Adaptive Control, 2nd Edition, Addison-Wesley, New York.

Bar-Shalom, Y., 1981, "Stochastic Dynamic Programming: Caution and Probing," IEEE Transactions on Automatic Control, Vol. AC-26, No. 5, pp. 1184-1195.

Bar-Shalom, Y., and Tse, E., 1974, "Dual Effect, Certainty Equivalence, and Separation in Stochastic Control," IEEE Transactions on Automatic Control, Vol. AC-19, No. 5, pp. 494-500.

De Keyser, R. M. C., Van De Velde, G. A., and DuMortier, F. A. G., 1988, "A Comparative Study of Self-Adaptive Long-range Predictive Control Methods,' Automatica, Vol. 24, pp. 149-163.

Hughes, D. J., and Jacobs, O. L. R., 1973, "Performance of LQG Control Sys tems Using Optimal k-step-ahead Control Laws," Proceedings of the Joint Automatic Control Conference, Columbus, OH., paper 22.4

Jacobs, O. L. R., and Patchell, J. W., 1972, "Caution and Probing in Stochastic Control," International Journal of Control, Vol. 16, No. 1, pp. 189-199.

$\mathrm{Ku}, \mathrm{R}$, and Athans, M., 1973, "On the Adaptive Control of Linear Systems Using the Open-Loop-Feedback-Optimal Approach," IEEE Transactions on Automatic Control, Vol. AC-18, No. 5, pp. 489-493.

Ljung, L., and Soderstrom, T., 1983, Theory and Practice of Recursive Identifcation, The MIT Press, Cambridge, MA.

Milito, R., Padilla, C. S., Padilla, R. A., and Cadorin, D., 1980, "Dual Control Through Innovations," IEEE Conference on Decision and Control, Vol. 1, pp. $341-345$.

Mookerjee, P., and Bar-Shalom, Y., 1989, "An Adaptive Dual Controller for a MIMO-ARMA System," IEEE Transactions on Automatic Control, Vol, 34 No. 7, pp. 795-800.

Sternby, J., 1976, "A Simple Dual Control Problem with an Analytical Solution," IEEE Transactions on Automatic Control, Vol. AC-21, No. 6, pp. 840-844.

Sternby, J., 1979, "Performance Limits in Adaptive Control," IEEE Transactions on Automatic Control, Vol. AC-24, No. 4, pp. 645-647.

Tse, E., and Bar-Shalom, Y., 1973, "An Actively Adaptive Control for Linear Systems with Random Parameters via the Dual Control Approach," IEEE Transactions on Automatic Control, Vol. AC-18, No. 2, pp. 109-117.

Wittenmark, B., 1975, "An Active Suboptimal Dual Controller for Systems with Stochastic Parameters," Automatic Control Theory and Applications, Vol. 3, No. 1, pp. 13-19.

\section{State Observer for Linear Time- Invariant Systems With Quantized Output}

\section{Joono Sur ${ }^{1}$ and Brad E. Paden ${ }^{2}$}

In this paper we introduce a state observer for linear timeinvariant systems with quantized outputs. The observer employs

\footnotetext{
'Department of Mechanical and Environmental Engineering, University of California, Santa Barbara, CA 93106.

${ }^{2}$ Associate Professor, Department of Mechanical and Environmental Engineering, University of California, Santa Barbara, CA 93106.

Contributed by the Dynamic Systems and Control Division of THE AMERICAN Society of Mechanical Engineers. Manuscript received by the Dynamic Systems and Control Division December 18, 1995. Associate Technical Editor: S. D. Fassois
}

an orthogonal projection operation at quantizer output discontinuities to enhance its convergence rate for stable systems. The increasing rate of convergence and stability has been proven by using Lyapunov second method. Some sufficient and necessary conditions of stability for the unstable systems are derived. The sufficient condition of noise stability is given and the maximal bound of noise stability is presented. The proposed methodology has been applied to state estimation of a DC-motor with optical encoder.

\section{Introduction}

In all digitally controlled plants, measured outputs are quantized prior to control computation. In most cases, the quantization error is small compared to system noise and is justifiably ignored. There are exceptions, however. One notable example is motor control where an optical encoder provides the only measured output, and mechanical position noise of the shaft due to vibration etc. is small as compared to quantization errors. In this paper we address the observer design problem for such linear time-invariant systems with quantized outputs. We show that incorporation of knowledge of the quantization nonlinearity leads to an improvement in the state estimate with a minor increase in observer complexity. Quantization has been addressed in the control systems context by several researchers. Curry (1970) has developed maximum likehood estimates for static linear systems driven by Gaussian noise and having quantized outputs. The extension to linear dynamic systems appears intractable analytically, however, Curry does derive approximate formulae for state estimates that work well with small quantizer steps. Another approach proposed by Schewppe (1968) propagates an ellipsoidal set which approximates the true system state by containment. This method only requires knowledge of bounds on inputs, and bounds on output measurement (quantizer) error; the performance calculation is intractable analytically in this case also. More recently, Miller et al. (1989) establish useful bounds on tracking performance in digitally controlled plants, where there is numerical quantization in the digital computation of the control input. Delchamp (1988) takes a new and fundamental approach to dealing with quantization. Rather than treating quantization as a bounded disturbance, his method treats quantization exactly, in the linear dynamic case, and establishes conditions under which the uncertainty in the system state tends to zero (as measured by differential entropy). The approach uses the system input to optimize information acquired on the state, but mixed approaches aimed at addressing information and tracking performance simultaneously now appear as possibilities. More recent works by Rotea and Williamson (1994) and others are representative of a broad class of problems focused on choosing state-space realization of discrete-time linear time-invariant systems which perform well in computer implementations. These methods effectively treat numerical round-off quantization as a noise source and address the scaling of internal signals to optimize competing objectives of $(a)$ low sensitivity to quantization and $(b)$ the desire for infrequent numerical overflow. The approach contrasts with the authors where quantization is modeled as a nonlinearity, rather than a noise source. Moreover, we necessary work with continuous systems rather than discrete-time systems. More recent work addressing chaos in feedback systems with quantization is due to Steppan and Haller (1996).

This paper has the following format. In Section 2 we motivate and introduce the observer of SISO and MIMO systems. In Section 3 we modify existing Lyapunov theory to accommodate the discontinuous updates used in our observer, and prove error convergence for a stable plant. Some sufficient and necessary conditions of stability for an unstable system are given. The maximal bound of noise stability is presented. Section 4 contains simulation results for the stable and unstable DC-motor with optical encoder. Our conclusions are made in Section 5. 